\title{
Experimental and numerical evidence of comparable levels of attenuation in periodic and a-periodic metastructures
}

\author{
J. M. De Ponti, ${ }^{1,2}$ (D) N. Paderno, ${ }^{1}$ (D) R. Ardito, ${ }^{1}$ (D) F. Braghin, ${ }^{2}$ (D) and A. Corigliano ${ }^{1, a)}$ (iD) \\ AFFILIATIONS \\ ${ }^{7}$ Department of Civil and Environmental Engineering, Politecnico di Milano, Piazza Leonardo da Vinci, 32,20133 Milano, Italy \\ ${ }^{2}$ Department of Mechanical Engineering, Politecnico di Milano, Via Giuseppe La Masa, 1, 20156 Milano, Italy \\ a)Electronic mail: alberto.corigliano@polimi.it
}

\begin{abstract}
"Phononic Crystals" are designed on the basis of a single cell periodically repeated in space along one or more directions. However, this may result as restrictive in terms of possible optimal solutions found. The present paper shows, numerically and experimentally, that it is possible to have high levels of attenuation also with a-periodic metastructures. Comparing two "phononic metastructures" externally identical but internally different, it is demonstrated that perturbing the periodicity inside the structure does not significantly affect its attenuation capabilities. This opens new possibilities in the field.
\end{abstract}

"Phononic Crystals" (PnCs) are important for their properties in mechanical wave filtering and control, related to their capability to show frequency bandgaps. ${ }^{1,2}$ Their study arose from electromagnetism. ${ }^{3-5}$ The performance of PnCs was mainly evaluated in terms of the bandgap width, with an optimization based on the dispersion spectrum of the unit cell. ${ }^{6}$ More recently, attention has been devoted to PnCs which are able to focus waves, exploiting lens or defected mode behaviors $^{7-12}$ also for energy harvesting purposes. ${ }^{13}$ In this prospective, topological metamaterials also seem to be very promising in wave guiding and confinement, ${ }^{14-16}$ reducing energy losses. In general, PnCs exhibit exceptional behaviors due to their periodic spatial arrangement of the building blocks, which causes, e.g., Bragg interference. ${ }^{2,5}$ Nevertheless, periodicity can be seen as a restrictive constraint; it is therefore interesting to discuss the consequences of a partial or total elimination of periodicity on the main performances of phononic metamaterials or structures captured by dispersion analysis and/or transmission properties. Dispersion analysis does not provide information about the bandgap dependence on the number and spatial distribution of unitary cells in a finite structure. ${ }^{17}$ It could happen that a bandgap existing in dispersion analysis does not exist in reality since more cells are needed. Conversely, transmission spectra provide a realistic structural response that can be directly compared with experimental results. The intrinsic nature of the bandgap in elastic metamaterials without a significant impedance mismatch has been demonstrated to rely on modal separation. ${ }^{18,19}$ The unit cell is able to exploit frequency gaps between low and high frequency modes. For these structures, bandgap maximization through dispersion analysis led to the definition of structures with a reduced number of modes (like a spring-mass chain) within a certain frequency range. However, looking at the transmission spectra, it is found that a-periodic structures are also able to exhibit high levels of attenuation, even if endowed with a higher number of modes. From an energetic point of view, this is associated with the capability of both structures to redistribute energy internally. The present work collates periodic and a-periodic metamaterials on the basis of numerical (FEM) and experimental evidences, mainly focussing on transmission spectra.

The a-periodic structure has been defined by perturbating an already existing periodic one ${ }^{18}$ (Fig. 1). Prototypes are built using Selective Laser Sintering (SLS) Additive Manufacturing (AM) technology. Both periodic and a-periodic structures define a slice with $15 \times 15 \mathrm{~cm}^{2}$ in-plane dimensions. Externally, the two structures are identical, with semispheres of the same dimensions, while internally, the a-periodic one is significantly perturbed with respect to the periodic one. The internal random perturbation preserves the same unit cell concept with four spheres attached on a frame. The details on geometry are reported in Table I with reference to the unit cell side length of $a=5 \mathrm{~cm}$. The adopted material is Nylon PA2200 (Young's modulus $E=1.70 \mathrm{GPa}$, Poisson's ratio $\nu=0.4$, density $\rho=925 \mathrm{~kg} / \mathrm{m}^{3}$, 


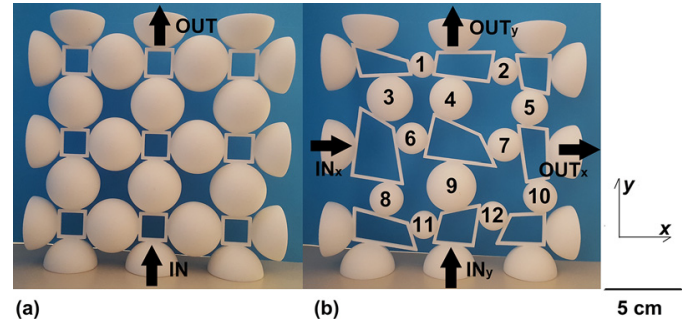

FIG. 1. Analyzed (a) periodic and (b) a-periodic structures obtained through SLS Additive Manufacturing technology.

TABLE I. Geometric parameters. $w_{\text {frame }}^{\text {in-pl. }}$ and $w_{\text {frame }}^{\text {out-pl. }}$ are the in-plane and out-ofplane frame thicknesses.

\begin{tabular}{lcc}
\hline \hline & Periodic [Fig. 1(a)] & A-periodic [Fig. 1(b)] \\
\hline $\boldsymbol{w}_{\text {frame }}^{\text {in-pl. }} \begin{array}{c}\text { out-pl. } \\
\boldsymbol{w}_{\text {frame }}\end{array}$ & $0.04 \mathrm{a}$ & $0.04 \mathrm{a}$ \\
$\boldsymbol{r}_{\text {sph }}$ & $0.05 \mathrm{a}$ & $0.05 \mathrm{a}$ \\
& $0.33 \mathrm{a}$ & (1) $0.152 \mathrm{a},(2) 0.167 \mathrm{a}$, \\
& (3) $0.279 \mathrm{a},(4) 0.265 \mathrm{a}$, \\
& (5) $0.223 \mathrm{a},(6) 0.197 \mathrm{a}$, \\
& (7) $0.210 \mathrm{a},(8) 0.207 \mathrm{a}$, \\
& (9) $0.311 \mathrm{a},(10) 0.208 \mathrm{a}$, \\
& (11) $0.173 \mathrm{a},(12) 0.180 \mathrm{a}$ \\
\hline \hline
\end{tabular}

and sound velocity $v=\sqrt{E / \rho}=1356 \mathrm{~m} / \mathrm{s}$ ). The two structures have a different total mass and stiffness, as shown in Table II.

The dynamic behavior of the periodic structure is described via a linear elastic dispersion analysis. The numerical band structure is calculated by means of ABAQUS and a proper implemented routine to apply Bloch-Floquet boundary conditions. The dispersion spectrum of the periodic structure is reported in Fig. 2, where a very wide bandgap in the frequency range of $1.57-16.29 \mathrm{kHz}$ can be observed. The periodic and a-periodic structures are compared looking at their linear elastic numerical transmission spectra between the input and output points shown in Fig. 1.

Figure 3 shows the transmission plots in the range of $0-20 \mathrm{kHz}$ : both structures exhibit high attenuation properties in almost the same frequency range even though the a-periodic structure involves a lot of moderate peaks due to the presence of many local modes. It is worth noticing that attenuation gaps appear in the a-periodic structure at low frequencies (Fig. 4). The associated deformed configurations involve local movements of internal portions of the structure of both spheres and frames, with a Locally Resonant (L.R.) behavior. The proposed aperiodic structure seems to comply with modal separation, with the

TABLE II. Global values of stiffness and mass computed numerically.

\begin{tabular}{lccc}
\hline \hline Structure & $\boldsymbol{k}_{\text {glob. }}^{x}(\mathrm{kN} / \mathrm{m})$ & $\boldsymbol{k}_{\text {glob. }}^{y}(\mathrm{kN} / \mathrm{m})$ & $\boldsymbol{m}_{\text {glob. }}(\mathrm{g})$ \\
\hline Periodic & 95.5 & 95.5 & 316 \\
A-periodic & 30.2 & 28.8 & 174 \\
$\Delta \%_{100[1-(a-\text { per. } / \text { per. })]}$ & 68.4 & 69.8 & 44.9 \\
\hline \hline
\end{tabular}

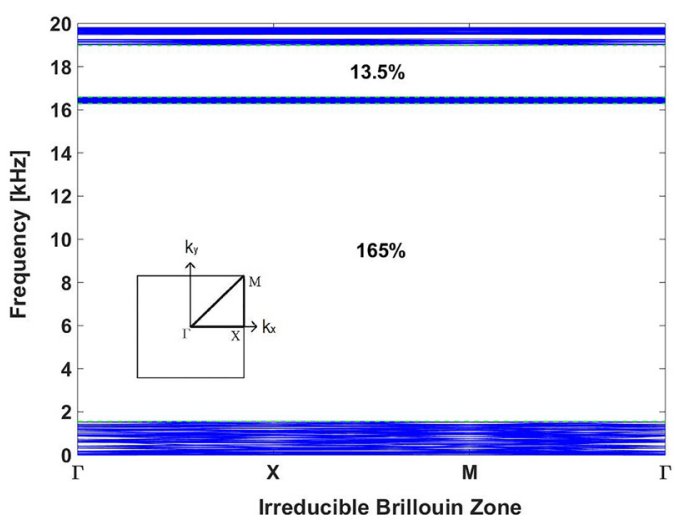

FIG. 2. Numerical band structure for the periodic structure. Bandgap width computed using the gap-midgap ratio.

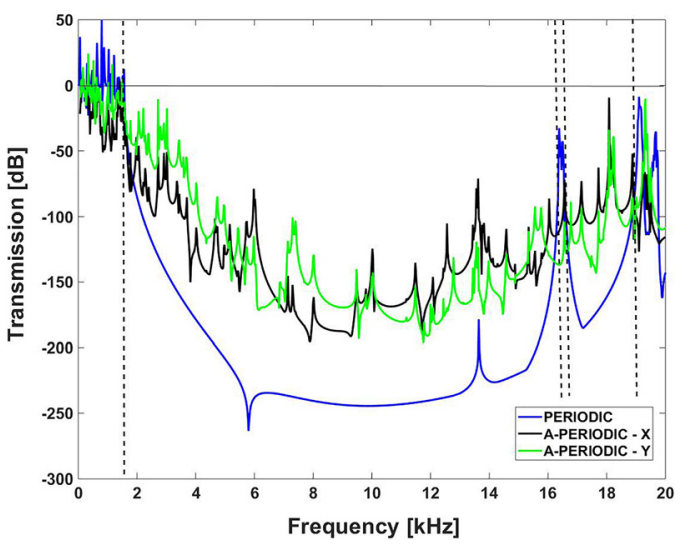

FIG. 3. Numerical transmission spectra in the frequency range of $0-20 \mathrm{kHz}$.

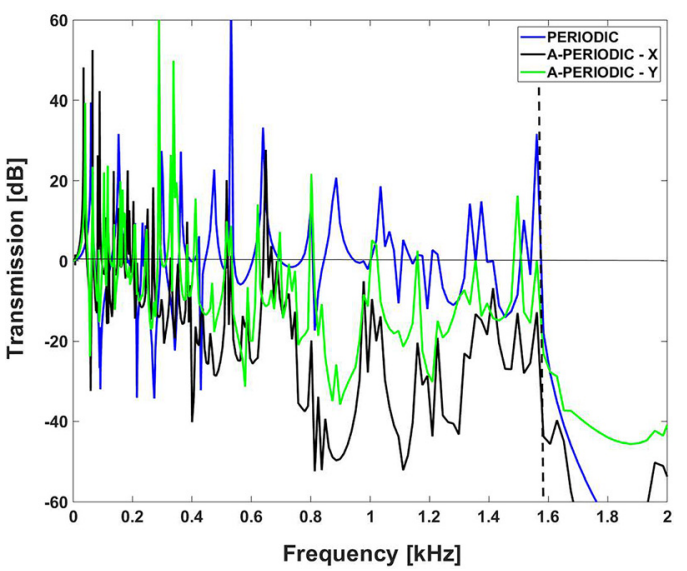

FIG. 4. Details of numerical transmission spectra in the frequency range of $0-2 \mathrm{kHz}$.

addition of L.R. disturbances in the whole frequency range. In the lowfrequency regime, the L.R. behavior entails peaks with nonevanescent transmission; conversely, in the rest of the spectrum, the L.R. peaks do not alter significantly the attenuation pattern. Such a behavior can be 
interpreted using simple spring-mass chain models, which, even if 1D, are able to catch the main features of this kind of $3 \mathrm{D}$ structure $^{18}$ for unidirectional input.

Let us consider a structure with global mass $m$ and global stiffness $k$ subject to a generic force $F(t)$. The simplest case is given by a structure composed of two point masses interconnected with a spring. The equations of motions for this system are

$$
\left\{\begin{array}{l}
\frac{m}{2} \ddot{u}_{1}(t)+k\left(u_{1}(t)-u_{2}(t)\right)=F(t), \\
\frac{m}{2} \ddot{u}_{2}(t)+k\left(u_{2}(t)-u_{1}(t)\right)=0 .
\end{array}\right.
$$

Applying a harmonic force $F(t)=\tilde{F} e^{i \omega t}$, in the steady state regime, the response also becomes harmonic in the form of $u_{i}(t)=\tilde{u}_{i} e^{i \omega t}$. Computing from the second of Eq. (1), the ratio $\left|\tilde{u}_{2}\right| /\left|\tilde{u}_{1}\right|$, the following is obtained:

$$
\left|\frac{\tilde{u}_{2}}{\tilde{u}_{1}}\right|=\left|\frac{k}{-\frac{m}{2} \omega^{2}+k}\right|=\left|\frac{1}{1-\beta^{2}}\right|=N(\beta),
$$

where $\beta=\omega / \omega_{0}$, with $\omega_{0}=\sqrt{2 k / m}$. $N(\beta)$ represents the wellknown magnification factor for an undamped single degree of freedom (SDOF) system under a harmonic force. It is clear that the structure starts to provide attenuation if $\beta>\sqrt{2}$, i.e.,

$$
\omega_{\text {att. }}=\sqrt{2} \sqrt{\frac{2 k}{m}}=2 \sqrt{\frac{k}{m}} .
$$

A finite periodic chain is obtained by repeating $(n-1)$ times the simple cell considered so far. If $\bar{m}$ and $\bar{k}$ denote the mass and stiffness of each cell, respectively, then the overall mass is $m=(n-1) \bar{m}$ and the overall stiffness is $k=\bar{k} /(n-1)$. It is well known ${ }^{1,2}$ that, for such a chain, the bandgap opens for $\omega_{\text {open }}^{B G}=2 \sqrt{\bar{k} / \bar{m}}$, which is in agreement with the previous calculation of $\omega_{\text {att }}$. The addition of cells does not alter the bandgap but creates other modes that are always located at a position below $\beta=\sqrt{2}$. Furthermore, whichever is the number of cells, the Frequency Response Function (FRF) takes the value of one for $\beta=\sqrt{2}$. It is possible to select the number of cells in order to have the same overall mass $m$, stiffness $k$, and bandgap opening frequency $\omega_{\text {open }}^{B G}$ as the numerically studied structure,

$$
n=1+\frac{\omega_{\text {open }}^{B G}}{2} \sqrt{\frac{m}{k}} .
$$

Solving Eq. (4), a value of almost 9 cells is obtained, which means 10 masses (with the first and the last being equal to $\bar{m} / 2$ ) and 9 springs, with $\bar{m}=35.1 \mathrm{~g}$ and $\bar{k}=859.5 \mathrm{kN} / \mathrm{m}$. In this way, the structure is modeled with an equivalent $1 \mathrm{D}$ chain (in terms of global mass and stiffness) able to correctly predict bandgap opening and the associated level of attenuation.

The most general case is the one in which the masses and stiffnesses are randomly distributed along the chain, i.e., without periodicity. Several works have been done for the characterization of a-periodic chains, ${ }^{20,21}$ mainly based on the Anderson theory of localization. The global response is generated by the combination of the responses of structural subelements that can be in the attenuation or amplification regime. A random spring mass chain is composed of $n$ different masses and $(n-1)$ different springs in series. The total mass and stiffness read as $m \equiv \sum_{i=1}^{n} m_{i} \quad k \equiv\left[\sum_{j=1}^{n-1} \frac{1}{k_{j}}\right]^{-1}$. In addition, it is possible to define the mean values $\langle m\rangle \equiv[1 / n] \sum_{i=1}^{n} m_{i}>0,\langle k\rangle$ $\equiv[1 /(n-1)] \sum_{j=1}^{n-1} k_{j}>0$ and the variances $\left\langle m^{2}\right\rangle \equiv[1 / n]$ $\sum_{i=1}^{n}\left(m_{i}-\langle m\rangle\right)^{2}, \quad\left\langle k^{2}\right\rangle \equiv[1 /(n-1)] \sum_{j=1}^{n-1}\left(k_{j}-\langle k\rangle\right)^{2}$. Two random distributions of mass and stiffness are generated, according to the following criteria: (i) the overall mass is the same as for the aperiodic structure sketched in Fig. 1(b); (ii) the global stiffness is the same as in the a-periodic structure, in the $x$ and $y$ directions, respectively; (iii) the highest natural frequency among the cells in the random chain is the same as the frequency of the single cell in the periodic chain. One finds $m_{i x}=(23.37,18.54,20.72,21.79,11.38$, $14.74,7.02,19.24,17.30,19.90) \mathrm{g}, m_{i y}=(20.36,21.15,17.93,21.62$, $20.50,14.33,6.44,20.59,12.93,18.15) \mathrm{g}, k_{j x}=(242.774,317.571$, 296.781, 253.254, 245.989, 242.612, 313.606, 273.034, 286.678) kN/m, and $k_{j y}=(252.805,244.330,310.796,251.729,259.326,259.568,248.046$, $240.959,278.287) \mathrm{kN} / \mathrm{m}$. The comparison between 3D a-periodic real structure and 1D models, in terms of global mass and stiffness and relevant standard deviations, is reported in Table III. The periodic and aperiodic structures exhibit approximately the same position of the attenuation band, as shown in Fig. 5. The a-periodic structure starts to attenuate before the periodic one, as also indicated by numerical simulations, due to the lower global stiffness. The attenuation pattern for the aperiodic structure begins around $1 \mathrm{kHz}$ : for the sake of comparison, if we consider the dispersion behavior for a periodic chain that includes the lowest stiffness and the largest mass, we found that the bandgap should open for $f_{\text {open }, x}^{B G}=2 \sqrt{242774 / 0.02337} / 2 \pi=1.03 \mathrm{kHz}$ and $f_{\text {open }, y}^{B G}=2 \sqrt{240959 / 0.02162} / 2 \pi=1.06 \mathrm{kHz}$. In the range between $1 \mathrm{kHz}$ and $1.5 \mathrm{kHz}$, the attenuation trend is interrupted by L.R. peaks, connected to the transmission modes of the random cells.

This kind of model, even if extremely simplified, is able to correctly predict the bandgap opening and shows clearly that it is possible to define a-periodic distributions with attenuation properties comparable to those of the periodic ones. This is quite important since it provides more freedom in the design of this kind of metastructure with respect to just considering the periodic ones. To further verify this interpretation, other configurations are considered, with slight changes in the random geometry of some cells (see Fig. 6 and Table IV). The

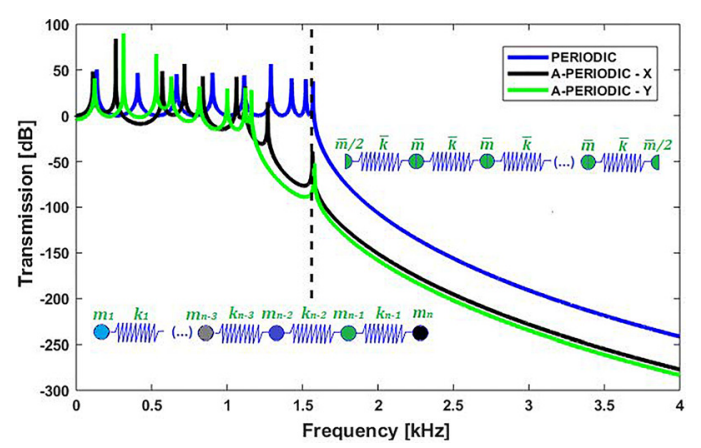

FIG. 5. Analytical transmission spectra of periodic and a-periodic spring-mass chains with a similar attenuation band position. 
TABLE III. Comparison between the real structure and the 1D model (in terms of mass and stiffness).

\begin{tabular}{lccc}
\hline \hline & Real structure & $1 \mathrm{D}-\mathrm{x}$ & $1 \mathrm{D}-\mathrm{y}$ \\
\hline$m_{\text {glob. }}(g)$ & 174.0 & 174.0 & 174.0 \\
$\sqrt{\left\langle m^{2}\right\rangle}(g)$ & 3.167 & 5.033 & 4.840 \\
$k_{\text {glob. }}^{x}(\mathrm{kN} / \mathrm{m})$ & 30.20 & 30.20 & $\ldots$ \\
$k_{\text {glob. }}^{y}(\mathrm{kN} / \mathrm{m})$ & 28.80 & $\ldots$ & 28.80 \\
$\sqrt{\left\langle k_{x}^{2}\right\rangle}(\mathrm{kN} / \mathrm{m})$ & 46.61 & 30.25 & $\ldots$ \\
$\sqrt{\left\langle k_{y}^{2}\right\rangle(\mathrm{kN} / \mathrm{m})}$ & 33.33 & $\ldots$ & 21.75 \\
\hline \hline
\end{tabular}

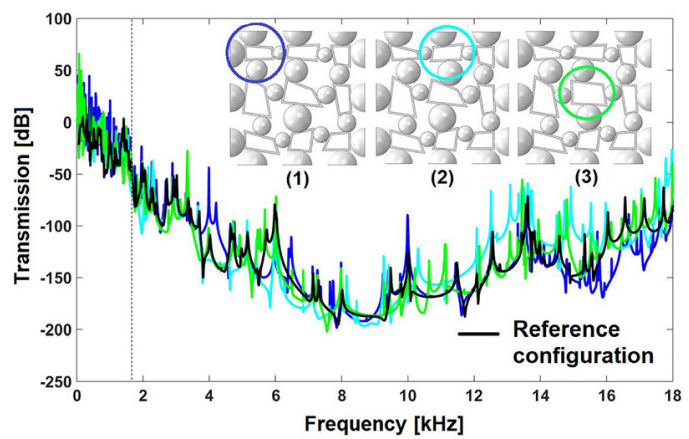

FIG. 6. Numerical transmission spectra (x-direction) when small perturbations are introduced in the a-periodic structure.

TABLE IV. Global values of stiffness and mass when small perturbations are introduced in the a-periodic structure.

\begin{tabular}{lcc}
\hline \hline Structure & $\boldsymbol{k}_{\text {glob. }}^{x}(\mathrm{kN} / \mathrm{m})$ & $\boldsymbol{m}_{\text {glob. }}(\mathrm{g})$ \\
\hline 1 & 30.0 & 170 \\
2 & 34.2 & 177 \\
3 & 29.3 & 170 \\
Reference & 30.2 & 174 \\
\hline \hline
\end{tabular}

analyses confirm that these randomly modified a-periodic structures provide attenuation in the same band as for the previous case (Fig. 6).

Experimental tests were carried out in order to validate numerical results. Prototypes were built using SLS Additive Manufacturing technology. The experimental setup is composed of an inertial shaker LDS v406 connected to a PA 100E Power Amplifier and two PCB Piezotronics 353B15 accelerometers, with a sensitivity of $10 \mathrm{mV} / \mathrm{g}$ and a resonance frequency of $70 \mathrm{kHz}$. A white noise excitation with a frequency range of $20 \mathrm{~Hz}-9 \mathrm{kHz}$ is imposed to the shaker. The input acceleration is measured using an accelerometer glued between the prototype and the shaker connection, while the output acceleration is measured at the opposite face of the periodic/a-periodic structure. The structure is placed on a very soft rubber foam, to avoid disturbances between input and output (Fig. 7).

Data acquisition is performed using NiMax Measurement Automatic explorer and postprocessed by means of a user Matlab

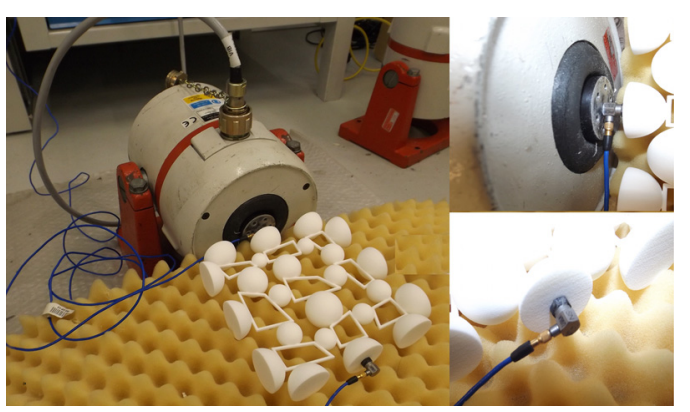

FIG. 7. Experimental setup and details of the accelerometers glued at the center of the top and bottom faces.

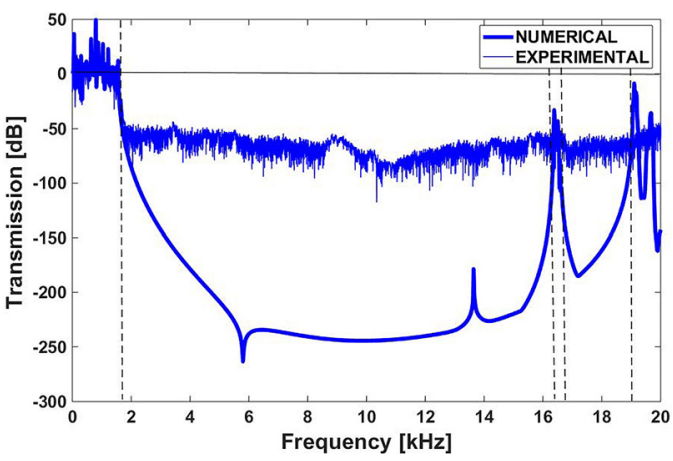

FIG. 8. Numerical and experimental transmission spectra of the periodic structure.

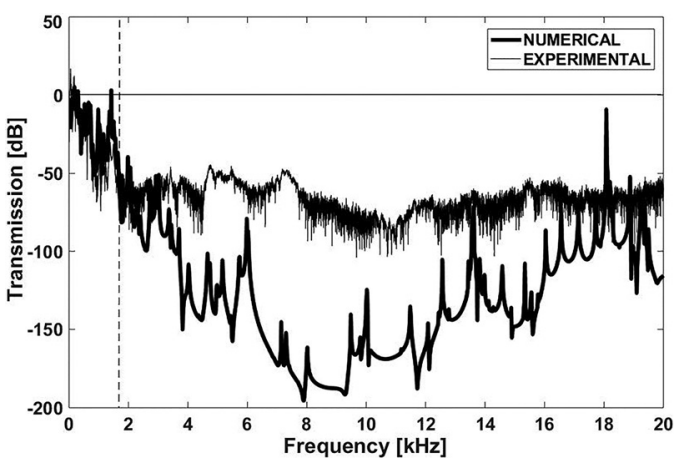

FIG. 9. Numerical and experimental transmission spectra of the a-periodic structure (x-direction).

routine. In Figs. 8, 9, and 10, numerical and experimental results are compared. Experimental results are in good agreement with numerical simulations, confirming the high attenuation capabilities of both periodic and a-periodic structures. Differences in attenuation are related to the accelerometer sensitivity, which provides a cut-off level of attenuation at around $-60 \mathrm{~dB}$. In Fig. 11, numerical and experimental transmission spectra are compared in the frequency range of $0-2 \mathrm{kHz}$, as previously done for numerical results only (Fig. 4). The examination of the results in the low-frequency regime confirms that the periodic structure shows an almost continuous transmission until the abrupt reduction in correspondence with the bandgap opening. On the other 


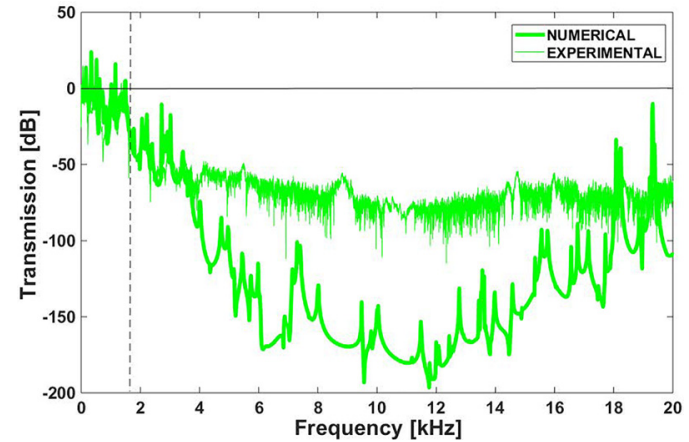

FIG. 10. Numerical and experimental transmission spectra of the a-periodic structure (y-direction).

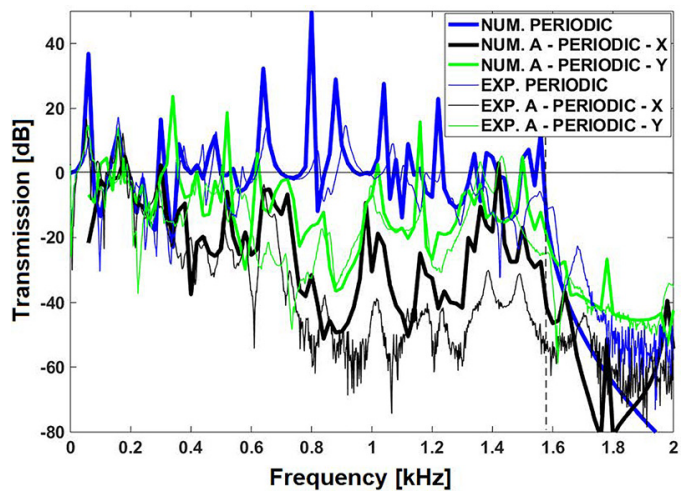

FIG. 11. Details of numerical and experimental transmission spectra.

hand, the a-periodic structure starts to attenuate earlier, as expected in view of the small overall stiffness, but several peaks are experimentally denoted, especially for the $y$ direction. This is in agreement with the numerical results and with the theoretical prediction for random spring-mass chains.

In this work, periodic and a-periodic metastructures have been shown to offer high levels of attenuation in the transmission spectrum when a proper separation of masses and stiffnesses is introduced inside the structure, which allows us to redistribute internally the total energy given to the system. Important parameters have been identified in the mass and stiffness of the finite structure and the dynamics of the single cells, which govern the main attenuation-band opening: it is important to remark that the proposed a-periodic structure provides the same level of attenuation of the periodic one with a lower global stiffness. Other attenuation bands can be created if different masses are introduced, due to L.R. phenomena. This interpretation can be used to design new metastructures able to show enhanced multifunctional behaviors. Such metastructures could be used for vibration insulation or noise suppression, with layouts that can be easily manufactured because of robustness with respect to imperfections and the lack of periodicity.
A.C. acknowledges the contribution of MIUR Project No. PRIN15 2015LYYXA8. J.M.D.P. acknowledges Politecnico di Milano for his Ph.D. grant on Smart Materials and Metamaterials for industry 4.0 .

\section{REFERENCES}

${ }^{1}$ L. Brillouin, Wave Propagation in Periodic Structures: Electric Filters and Crystal Lattices (Dover Publications, Inc., New York, 1946).

${ }^{2}$ V. Laude, Phononic Crystals: Artificial Crystals for Sonic, Acoustic, and Elastic Waves (De Gruyter, 2015).

${ }^{3}$ C. Kittel and B. C. Kittel, Introduction to Solid State Physics (Wiley, New York, 1976).

${ }^{4}$ N. W. Ashcroft and N. D. Merim, Solid State Physics (Harcourt College Publishers, USA, 1976).

${ }^{5}$ M. Hussein, M. Leamy, and M. Ruzzene, "Dynamics of phononic materials and structures: Historical origins, recent progress, and future outlook," Appl. Mech. Rev. 66, 040802 (2014).

${ }^{6}$ L. D’Alessandro, B. Bahr, L. Daniel, D. Weinstein, and R. Ardito, "Shape optimization of solid-air porous phononic crystal slabs with widest full 3D bandgap for in-plane acoustic waves," J. Comput. Phys. 344, 465-484 (2017).

${ }^{7}$ S. Tol, F. L. Degertekin, and A. Erturk, "Phononic crystal Luneburg lens for omnidirectional elastic wave focusing and energy harvesting," Appl. Phys. Lett. 111, 013503 (2017).

${ }^{8}$ M. Kurosu, D. Hatanaka, K. Onomitsu, and H. Yamaguchi, "On-chip temporal focusing of elastic waves in a phononic crystal waveguide," Nat. Commun. 9, 1331 (2018).

${ }^{9}$ M. G. Baboly, C. M. Reinke, B. A. Griffin, I. El-Kady, and Z. C. Leseman, "Acoustic waveguiding in a silicon carbide phononic crystals at microwave frequencies,” Appl. Phys. Lett. 112, 103504 (2018).

${ }^{10}$ S. Tol, F. L. Degertekin, and A. Erturk, "3D-printed lens for structure-borne wave focusing and energy harvesting," Proceedings of SPIE 10164, Active and Passive Smart Structures and Integrated Systems (2017).

${ }^{11}$ X. Yan, R. Zhu, G. Huang, and F. G. Yuan, "Focusing guided waves using surface bonded elastic metamaterials,” Appl. Phys. Lett. 103(12), 121901 (2013).

${ }^{12}$ T. T. Wu, Y. T. Chen, J. H. Sun, S. C. S. Lin, and T. J. Huang, "Focusing of the lowest antisymmetric Lamb wave in a gradient-index phononic crystal plate," Appl. Phys. Lett. 98(17), 171911 (2011).

${ }^{13}$ M. Carrara, M. Cacan, J. Toussaint, M. Leamy, M. Ruzzene, and A. Erturk, "Metamaterial-inspired structures and concepts for elastoacoustic wave energy harvesting," Smart Mater. Struct. 22, 065004 (2013).

${ }^{14} \mathrm{~T}$. Liu and F. Semperlotti, "Experimental evidence of robust acoustic valley Hall edge states in a non-resonant topological elastic waveguide," Phys. Rev. Appl. 11, 014040 (2019).

${ }^{15}$ M. P. Makwana and R. V. Craster, "Designing multidirectional energy splitters and topological valley supernetworks,” Phys. Rev. B 98, 235125 (2018).

${ }^{16}$ A. Khelif, M. Wilm, V. Laude, S. Ballandras, and B. Djafari-Rouhani, "Guided elastic waves along a rod-defect of a two-dimensional phononic crystal," Phys. Rev. E 69, 067601 (2004).

${ }^{17}$ C. Sugino, Y. Xia, S. Leadenham, M. Ruzzene, and A. Erturk, "A general theory for bandgap estimation in locally resonant metastructures," J. Sound Vib. 406, 104-123 (2017).

${ }^{18}$ L. D’Alessandro, E. Belloni, R. Ardito, A. Corigliano, and F. Braghin, "Modeling and experimental verification of an ultra-wide bandgap in 3D phononic crystal," Appl. Phys. Lett. 109, 221907 (2016).

${ }^{19}$ L. D'Alessandro, E. Belloni, R. Ardito, F. Braghin, and A. Corigliano, "Mechanical low-frequency filter via modes separation in $3 \mathrm{D}$ periodic structures," Appl. Phys. Lett. 111, 231902 (2017).

${ }^{20}$ F. A. B. F. de Moura, L. P. Viana, and A. C. Frery, "Vibration modes in aperiodic one-dimensional harmonic chains,” Phys. Rev. B 73, 212302 (2006).

${ }^{21}$ S. Russ, "Scaling of the localization length in linear electronic and vibrational systems with long-range correlated disorder," Phys. Rev. B 66, 012204 (2002). 